\title{
A Visual Servoing Method For High Precision Chip Mounting Technology
}

\author{
Duk-Young Lee, Kyung-Nam Jang and Hyungsuck Cho \\ Dept. of Mechanical Eng., Korea Advanced Institute of Science and Technology
}

\begin{abstract}
This paper presents a visual servoing methodology to realize a vision-based chip mounting, which makes it possible to mount micro chips to PCB with high precision. Because of continued down-sizing of electronic products, density of electronic parts to be packed into PCBs is ever getting higher and higher, thus requiring surface mounting technologies (SMT) to be more precise and productive. Down-sizing also tends toward miniaturization of components. The increased package density and component miniaturization require not only delicate handling of small chips but also delicate placement of them on PCBs. In order to place the small chips on the pattern of PCB, highly accurate mounting process is ultimately required. This is because it is difficult to place the chips a specified position with tiny small contact force due to uncertainty involved with the kinematic calibration error of the mounter and the deformation of PCB. In this paper, to avoid this difficulty we propose a visual servoing method for the chip mounting system. In such a way, the proposed visual servoing technique guarantees that chips are accurately placed at the desired positions. To achieve this, a CCD camera views the chip mounted with the mark on PCB simultaneously, and then the edge features and corner features are extracted from the acquired image. The proposed visual servoing algorithm generates the motion of the mounter to align the features of the chip with the ones of the mark on PCB on-line. The result obtained from real time experiment is discussed from the view points of practicality of the proposed method.
\end{abstract}

Keywords : Surface Mounting Technology, Visual Servoing, Feature Extraction, Feature Tracking, Occlusion.

\section{INTRODUCTION}

In recent years, the global trend for more functionality, better enery efficiency and a high reliability is the driving factor for the introduction of micro parts. Dramatic improvement in performance of microprocessors has resulted in a huge supply of personal computer. Moreover, he demand of network service in telecommunication and distributed computers such as the Internet has increased, so that the fiber optic network market is growing very rapidly driven by increased demand for bandwidth for data communication. Fiber optic networks are being installed because they provided greater capacity, more cost effectively than traditional copper wire based networks. Network growth is driving similar high growth rates for optical components but it is difficult to implement automation and scale manufacturing because of requiring the precision of micro scale. A mobile phone in human life is significant personal communication. It includes many functions such as the camera, personal computer and game etc., and its size become smaller. Thus, the micro electronic part such as bare chip is required[1]. A demand for miniaturized systems in various applications is observed in recent years. The trend to higher integration in microelectronics and the integration of micromechanical, microfluidic and microoptical components in mainstream applications requires a new type of microsystem. The micro parts require the handling system such as automatic assembly. The handling system with submicron precision in the placement of parts is beyond the calibration range of open loop precision assembly device used in industry. The use of vision sensors in feedback loop combined with high resolution optical systems achieves the required precision compensate for inaccurate sensor and kinematic error[2-4]. The visual servoing allows for the closed loop control of a end-effector through the use of the camera[2-6].

In this paper, we introduce the proposed chip mounting system[7]. Conventional chip mounting system uses the vision systems which are a device camera to observe the chip, and a fiducial camera to observes the fiducial mark on the PCB

E-mail : hscho@lca.kaist.ac.kr ; phone : +82-42-869-3253; fax +82-42-869-3210; http://lca.kaist.ac.kr ; Dept. of Mechanical Engineering, KAIST, 373-1, Guseong-dong, Yuseong-gu, Daejeon, Korea, 305-701. 
board. These cameras measure the relative pose of the PCB board using calibration information, and then both of them align no more camera feedback. We propose a chip mounting system with online feedback of the camera, and visual servoing algorithm. The camera can simultaneously observe the chip and the mark on the PCB. In this paper,. For experiment, we use the TR chip whose size is $2.0 \times 1.2 \mathrm{~mm}$.

\section{CHIP MOUNTING SYSTEM}

In the mounting process of the electronic parts, the components are placed onto the circuit pattern, which have the covering of solder paste. This process is called "on placement". After this process, the chip mounted PCB is soldered on the reflow oven. The mounting process is schematically shown in Figure 1, where multiple mounter heads are working for the placement task. Surface mounting of electronic parts is composed of four major processes. First the mounter head picks up an electronic chip. Then it is moved into the vision stage where identification and inspection of that is made by a device camera. PCB pose is measured by detecting fiducial marks with a fiducial camera. The chip is placed onto the desired location.

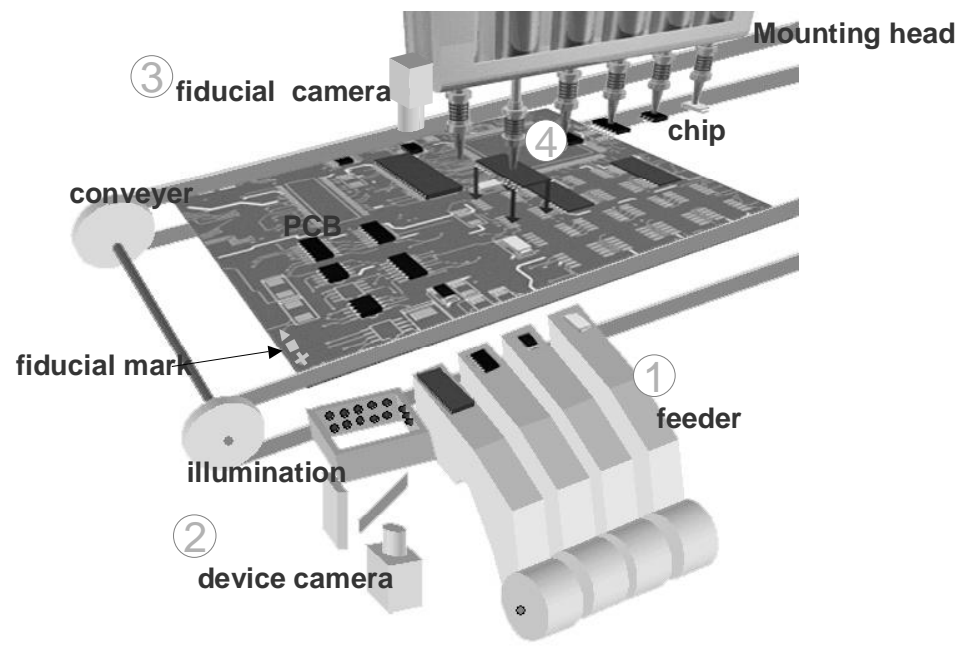

Figure 1: Conventional chip mounting System

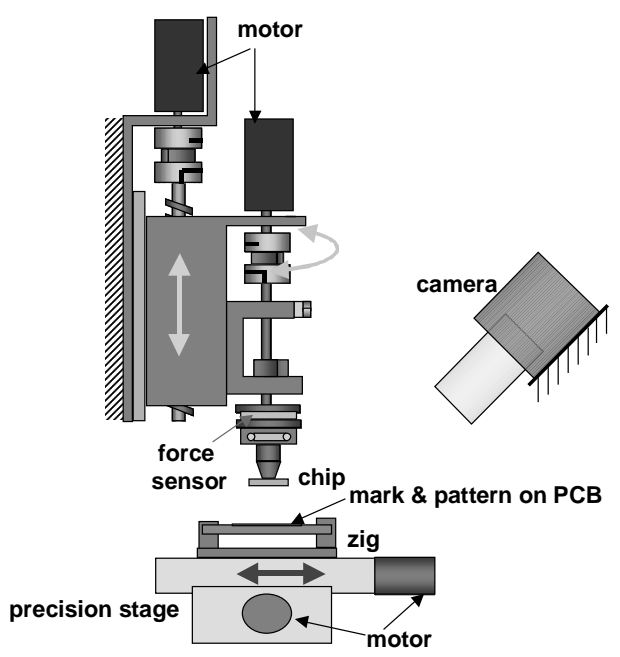

Figure 2: The proposed visual servoing system

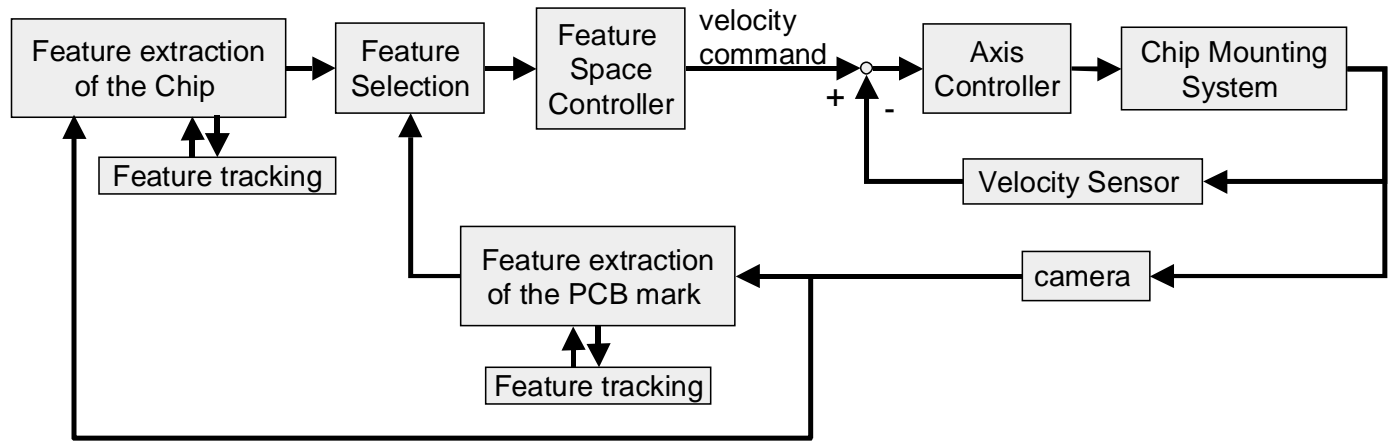

Figure 3: Schematic of the servoing system

Figure 2 shows the proposed chip mounting system equipped with a camera system. It consists of the driving systems and the visual feedback system. The driving systems are the chip mounting head and the x-y stage for moving the mark at the desired position. Chip mounting head consists of a macro ac servomotor with a ball screw mechanism and a rotational motor to align the orientation of the chips with the mark on PCB surface. Since the chips are passively 
aligned with the surface of PCB tilted very small angle, tilting motion is not considered in these mounting heads. A vacuum generator is utilized for the loading /unloading of the electronic parts at the mounting end tip, and a load cell is mounted to measure the contact force between the nozzle and the rotational shaft. The vision camera plays an important role in aligning the chip with the mark and gives the motion command to the controller by detecting and tracking the features of the chip and the mark. A focused position of the camera is located in the pattern and the mark of the substrate. As the chip approaches the mark that represents the desired position, it is clear that the chip occludes the mark. Therefore the occlusion problem is important to visual servong with microscopic view. Figure 3 shows the control system architecture. The features of the chip and the mark on the PCB are extracted from the image of the camera and are tracked by visual tracking algorithm. The features of the mark matched with feature of the chip are selected and the error of these features generates the motion command by visual servoing algorithm.

\section{FEATURE EXTRATION}

The chip mounting process needs to align the mounting chip with the mark on the PCB board. We should extract the features from the chip and the mark that correspond with each other. In this system, the bottom edge of the chip moves and aligns the inner boundary with the mark on the board. Therefore, the features should be defined and extracted by applying the image-processing algorithms to the image acquired in the camera. In the case of TR chip, the features are inside boundary of the mark and bottom edges of the chip. The correspondence of each other is decided by the position of the lead and the pattern shape.

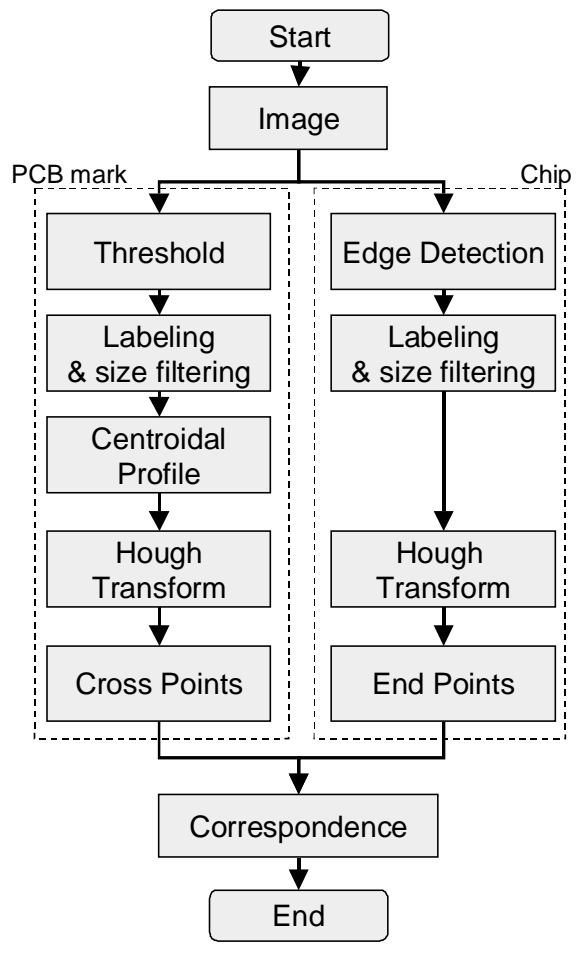

(a) Flow Chart of Feature Extraction

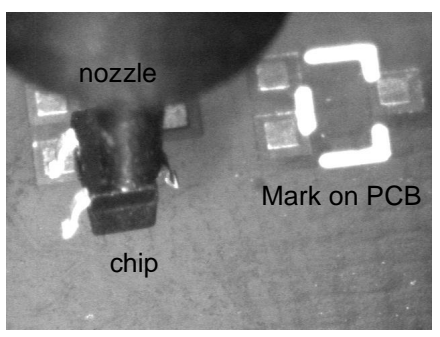

(b) Captured Image

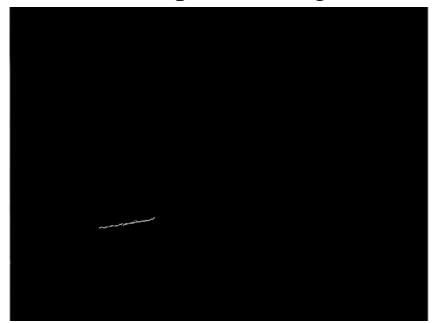

(d) Edge Detection

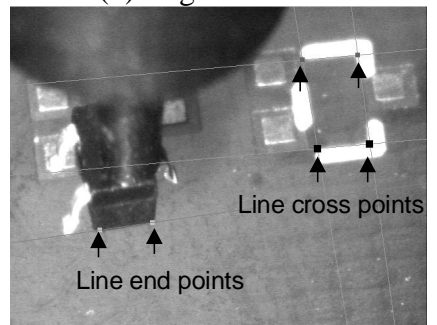

(f) Line extraction

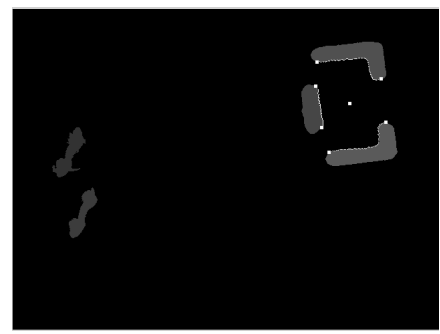

(c) Threshold \& Labeling

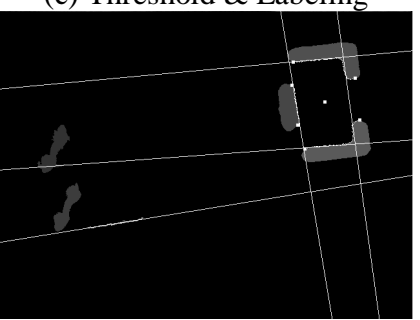

(e) Hough Transform

Figure 4: Feature Extraction

Figure 4(a) shows the flow chart for extracting the features of the chip and the mark on the PCB for the TR chip. Figure 4(b)-(f) illustrates the result images at the each process of the feature extraction. At first, Threshold is applied at the acquired image shown in Figure 4(b) in order to extract the lead of the chip and the mark. It is simple algorithm 
and defined as the following equation.

$$
I(u, v)=\left\{\begin{array}{lc}
1 & \text { if } \quad I(u, v)>T \\
0 & \text { otherwise }
\end{array}\right.
$$

where $I(u, v)$ denotes image intensity function at image pixel coordinate $(u, v)$ and $\mathrm{T}$ is the threshold value. Binary image through threshold classifies the bright area and the dark area. In this image, the lead of the chip and the mark belongs to the bright area.

Figure 4(c) shows the result image after labeling the binary image. Next the centroidal profile of three pieces at the binary image is calculated and Figure 5 shows the centroidal profile of the mark. The centroidal profile can be calculated by distance from the center point $\mathrm{C}$ to point that is on the inner boundary of the label data or on the scanning boundary in each direction. The scanning boundary is limited by square with 100-pixel distance from point $\mathrm{C}$. In Figure 5(b), the points between a and b, $\mathrm{c}$ and $\mathrm{d}$, and e and $\mathrm{f}$ represent the distance from center point to the point to meet the labeled points in $\phi$-direction, and are meaningful data. The others are points that meet the scan boundary, which is defined as square shown Figure 5(a). They are meaningless data. The shortest interval c-d of three groups can be classified with the others and that gives clue about the orientation of the mark.

Using Hough transform, which transforms image pixel coordinate $u-v$ transforms to the $\rho-\theta$ space, we can obtain the line parameter $\rho$ and $\theta$ from these meaningful data. The relationship between $u-v$ and $\rho-\theta$ is written as

$$
u \cos \theta+v \sin \theta=\rho
$$

The bottom edge of the chip is the points that change dark intensity to bright intensity. The following conditions by $v$ directional operator of Sobel operation and threshold technique can detect the bottom edge.

$$
\begin{aligned}
& I(u-1, v+1)+2 I(u, v+1)+I(u+1, v+1)-[I(u-1, v-1)+2 I(u, v-1)+I(u+1, v-1)]>T_{\text {sobel }} \\
& I(u, v)<T_{\text {chip }}
\end{aligned}
$$

where $T_{\text {sobel }}$ and $T_{\text {chip }}$ are threshold values. The line parameter is obtained from these points by using Hough Transform. The position and the number of the lead give a clue to decide the direction of the chip. The correspondence of the feature points can be decided from the direction of the mark and the chip.

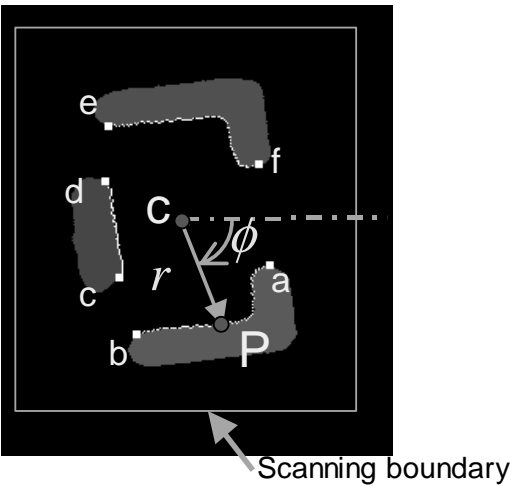

(a) Image

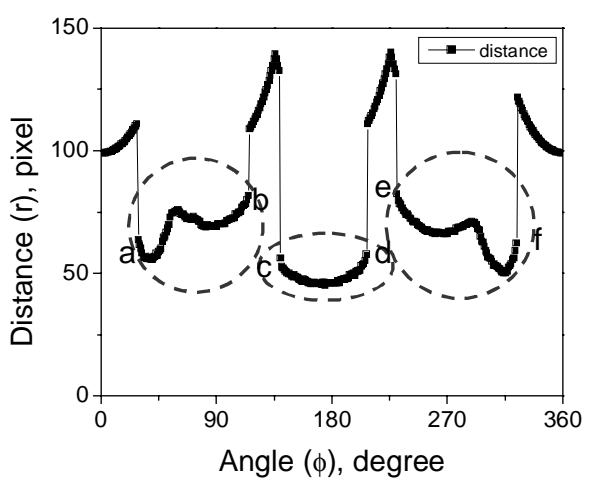

(b) Centroidal profile

Figure 5: Centroidal Profile 


\section{FEATURE TRACKING}

The feature extraction algorithm is complex. Thus, simple method is required to detect the position of feature points and find the correspondences in real time. Visual tracking is to find the correspondence of the feature points at the current image with at the previous image.

\subsection{Visual tracking and occlusion}

Sum-of-square-differences(SSD) tracking allows for tracking multiple features in real time due to the short processing time. It finds the displacement $\mathbf{d}=(\Delta u, \Delta v)^{T}$ that minimizes the SSD measure:

$$
e(\mathbf{d})=\sum_{m . n \in N}\left[I\left(u\left(t_{i-1}\right)+m, v\left(t_{i-1}\right)+n, t_{i-1}\right)-I\left(u\left(t_{i-1}\right)+m+\Delta u, v\left(t_{i-1}\right)+n+\Delta v, t_{i}\right)\right]^{2}
$$

where $N$ is an searching area around the pixel we are interested in, and $I\left(\cdot, t_{i}\right), I\left(\cdot, t_{i-1}\right)$ are the image intensity functions in time $t_{i}, t_{i-1}$, respectively. SSD has the minimum value in matching point. If the matching image is perfectly same, SSD value is zero.

$$
\min [e(\mathbf{d})]=0
$$

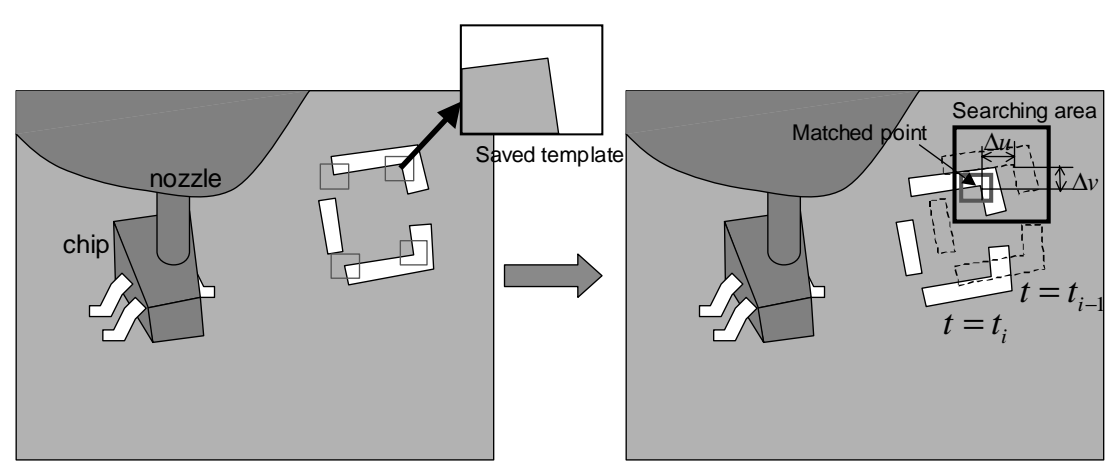

(a) Feature tracking

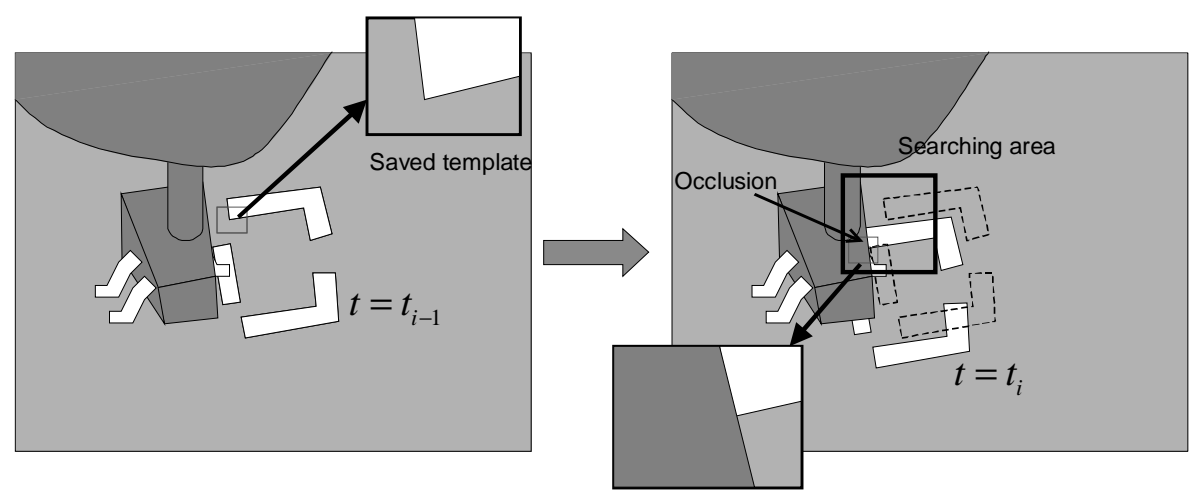

(b) Occlusion

Figure 6: Tracking and occlusion

When the occlusion of the features occurs, we should decide whether occlusion occurs or not. Because the occluded feature in the searching area has large minimum SSD value. Thus, the following equation can discriminate the 
occluded features from the shown features.

$$
\min [e(\mathbf{d})]>T_{o c}
$$

where, $T_{o c}$ is the threshold value for discrimination of the occlusion.

Figure 6 illustrates the example of the feature tracking and the occlusion. SSD saves image in the window for the extracted feature point as the template at time $t_{i-1}$, and then finds the position of image intensity matched with saved template in searching area as shown Figure 6(a). When occlusion occurs, the saved template is not matched in searching area and the minimum of SSD has large error.

\subsection{Position estimation of the occlusion}

When the occlusion occurs during tracking the features for visual servoing, its position should be estimated. Two clues for estimating its position are given. One is the geometry information of the features and the other is the motion information of the stage.

In this application four lines define the geometry of the mark, which does not rotate. The position of the occluded feature can be estimated by using the position $\left(u_{n i}\left(t_{i}\right), v_{n i}\left(t_{i}\right)\right)$ of the neighbor point $j$ and the orientation $\theta_{c n i}\left(t_{i-1}\right)$ of the line passing both of them at time $t_{i-1}$. Line equation crossing the neighbor point and the occluded feature can be written as

$$
u \cos \theta_{n j}\left(t_{i-1}\right)+v \sin \theta_{n j}\left(t_{i-1}\right)=\rho
$$

where $\rho=u_{n j}\left(t_{i}\right) \cos \theta_{n j}\left(t_{i-1}\right)+v_{n j}\left(t_{i}\right) \sin \theta_{n j}\left(t_{i-1}\right)$.
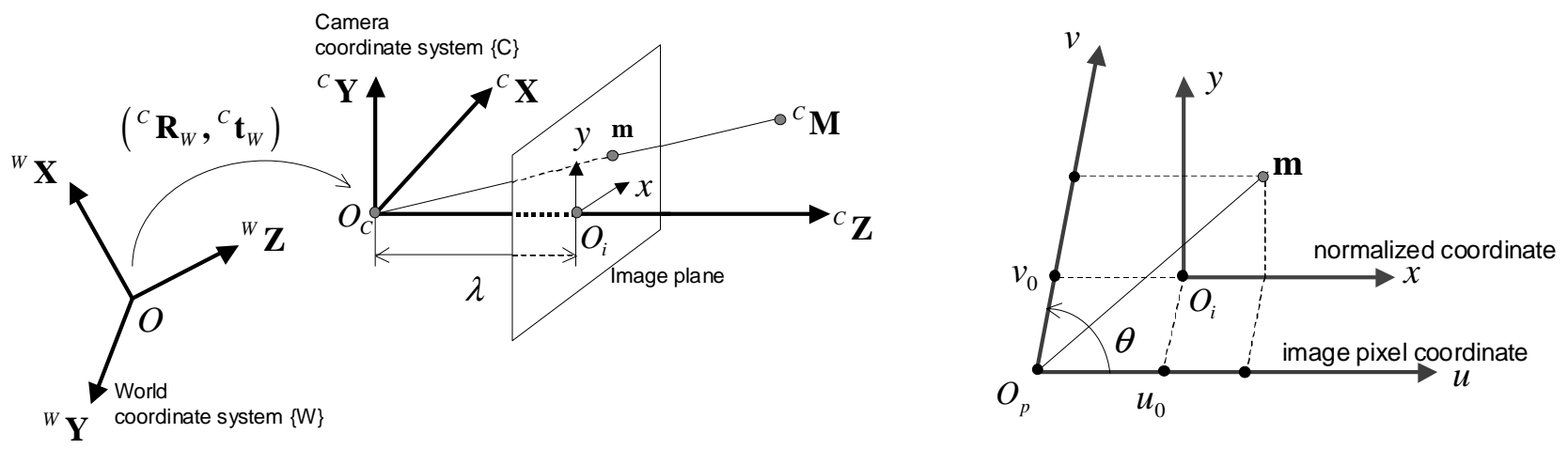

Figure 7: Camera model

The motion information of the stage gives the position of the features at the image space using the camera calibration information. This approach is influence by the calibration error.

From Figure 7 it should be clear that the relationship between 3 -D space coordinate ${ }^{C} \mathbf{M}=\left[\begin{array}{llll}{ }^{C} X & { }^{C} Y & { }^{C} Z\end{array}\right]^{T}$ and the normalized coordinate $\mathbf{m}=\left[\begin{array}{ll}x & y\end{array}\right]^{T}$ of the image plane at the CCD camera can be written as

$$
\begin{aligned}
& x=\lambda \frac{{ }^{C} X}{{ }^{C} Z} \\
& y=\lambda \frac{{ }^{C} Y}{{ }^{C} Z}
\end{aligned}
$$


which can be rewritten linearly as

$$
s\left[\begin{array}{c}
x \\
y \\
1
\end{array}\right]=\left[\begin{array}{cccc}
\lambda & 0 & 0 & 0 \\
0 & \lambda & 0 & 0 \\
0 & 0 & 1 & 0
\end{array}\right]\left[\begin{array}{c}
{ }^{C} X \\
{ }^{C} Y \\
{ }^{C} Z \\
1
\end{array}\right]=\mathbf{P}\left[\frac{{ }^{C} \mathbf{M}}{1}\right]
$$

where, $\lambda$ is the focal length of the camera and $s$ is arbitrary constant.

The relationship between the normalized coordinate and the pixel coordinate shown in Figure 7(b) can be written as

$$
\left[\begin{array}{l}
u \\
v \\
1
\end{array}\right]=\left[\begin{array}{ccc}
k_{u} & k_{v} \cot \theta & u_{0} \\
0 & k_{v} / \sin \theta & v_{0} \\
0 & 0 & 1
\end{array}\right]\left[\begin{array}{l}
x \\
y \\
1
\end{array}\right]=\mathbf{H}\left[\begin{array}{c}
\mathbf{m} \\
\frac{1}{1}
\end{array}\right]
$$

where $k_{u}$ and $k_{v}$ are the scaling factors along the $x$ and $y$ axes (in pixel/meter), $u_{0}$ and $v_{0}$ are the pixels coordinates of the principal point.

$$
s\left[\begin{array}{c}
u \\
v \\
1
\end{array}\right]=\left[\begin{array}{ccc}
\lambda k_{u} & \lambda k_{v} \cot \theta & u_{0} \\
0 & \lambda k_{v} / \sin \theta & v_{0} \\
0 & 0 & 1
\end{array}\right]\left[\begin{array}{c}
{ }^{C} X \\
{ }^{C} Y \\
{ }^{C} Z \\
1
\end{array}\right]=\mathbf{H P}\left[\frac{{ }^{C} \mathbf{M}}{1}\right]=\tilde{\mathbf{p}}\left[\frac{{ }^{C} \mathbf{M}}{1}\right]
$$

where $\tilde{\mathbf{P}}$ denotes the projective matrix. The parameters $\alpha_{u}=\lambda k_{u}, \alpha_{v}=\lambda k_{v}, \phi, u_{0}$ and $v_{0}$ do not depend on the position and orientation of the camera in space, and they are thus called intrinsic[6,8]. In Figure $7{ }^{C} \mathbf{R}_{W}$ and ${ }^{C} \mathbf{t}_{W}$ are the rotational matrix and translation matrix between the camera frame $\{C\}$ and the world frame $\{W\}$. The parameters of these transformation matrixes are called extrinsic. From equations (8)-(11) the relationship between the 3-D coordinate with respect to world frame $\{\mathrm{w}\}$ and the image pixel coordinate can be written as

$$
s\left[\begin{array}{c}
u \\
v \\
1
\end{array}\right]=\tilde{\mathbf{p}}\left[\begin{array}{c|c}
{ }^{C} \mathbf{R}_{W} & { }^{C} \mathbf{t}_{W} \\
\hline \mathbf{O}_{3}^{T} & 1
\end{array}\right]\left[\frac{{ }^{W} \mathbf{M}}{1}\right]=\tilde{\mathbf{P}}\left[\frac{{ }^{W} \mathbf{M}}{1}\right]
$$

where $\mathbf{O}_{3}^{T}=\left[\begin{array}{lll}0 & 0 & 0\end{array}\right]$. The camera calibration is the process of estimating the intrinsic and extrinsic parameters of a camera.

When the $X Y$ stage moves by $\Delta X$ and $\Delta Y$ with respect to stage frame $\{S\}$ from time $t_{i-1}$ to $t_{i}$, the position ${ }^{S} X\left(t_{i}\right)$, ${ }^{s} Y\left(t_{i}\right)$ of the occluded feature at time $t_{i}$ can written as

$$
\begin{aligned}
& { }^{s} X\left(t_{i}\right)={ }^{s} X\left(t_{i-1}\right)+\Delta X \\
& { }^{s} Y\left(t_{i}\right)={ }^{s} Y\left(t_{i-1}\right)+\Delta Y
\end{aligned}
$$

where ${ }^{S} X\left(t_{i-1}\right)$ and ${ }^{S} Y\left(t_{i-1}\right)$ denote its position at time $t_{i-1}$. 


$$
\left[\begin{array}{c}
{ }^{s} X\left(t_{i-1}\right) \\
{ }^{s} Y\left(t_{i-1}\right) \\
{ }^{s} Z\left(t_{i-1}\right) \\
1
\end{array}\right]=s\left[\begin{array}{l|l}
{ }^{C} \mathbf{R}_{S} & { }^{C} \mathbf{t}_{s} \\
\hline \mathbf{O}_{3}^{T} & 1
\end{array}\right]^{-1} \tilde{\mathbf{P}}^{+}\left[\begin{array}{c}
u\left(t_{i-1}\right) \\
v\left(t_{i-1}\right) \\
1
\end{array}\right]
$$

where ${ }^{S} Z\left(t_{i-1}\right)$ is known and constant because XY stage does not generate $\mathrm{Z}$ direction motion, and ${ }^{C} \mathbf{R}_{S}$ and ${ }^{C} \mathbf{t}_{S}$ are the relationship between the camera frame $\{\mathrm{C}\}$ and the stage frame $\{\mathrm{S}\}$.

Therefore, image pixel coordinate $\left(u\left(t_{i}\right), v_{k}\left(t_{i-1}\right)\right)$ of the occluded feature at time $t_{i}$ can derived as

$$
s\left[\begin{array}{c}
u\left(t_{i}\right) \\
v\left(t_{i}\right) \\
1
\end{array}\right]=\tilde{\mathbf{P}}\left[\begin{array}{c|c}
{ }^{C} \mathbf{R}_{S} & { }^{C} \mathbf{t}_{s} \\
\hline \mathbf{O}_{3}^{T} & 1
\end{array}\right]\left[\begin{array}{c}
{ }^{s} X\left(t_{i}\right) \\
{ }^{s} Y\left(t_{i}\right) \\
{ }^{s} Z\left(t_{i}\right) \\
1
\end{array}\right]
$$

where ${ }^{s} Z\left(t_{i}\right)={ }^{s} Z\left(t_{i-1}\right)$.

\section{VISUAL SERVOING EXPERIMENT}

Figure 8 shows the flow chart for mounting. The features and the correspondence can be extracted from the first captured image. In next steps, the features are tracked at the next captured images using SSD tracking method. The proposed servoing algorithm aligns the orientation of them and then the position in $x$ and $y$ direction. Finally, the proposed algorithm aligns the mark with the bottom edge of the chip while the mounting head move to downward.

\subsection{Visual servoing using line segments}

In order to align the chip with the mark on the PCB, features for visual servoing are the mid points and the orientation of line segments whose end points are tracked. The velocity $v_{x}$ and $v_{y}$ in $x$ and $y$ direction of the stage and rotational velocity $\omega$ of the mounting head can be derived as

$$
\begin{aligned}
& v_{x}=k_{x} \frac{\sum\left(f_{u, c i}-f_{u, m i}\right)}{n} \\
& v_{y}=k_{y} \frac{\sum_{i}^{n}\left(f_{v, c i}-f_{v, m i}\right)}{n} \\
& \omega=k_{\theta} \frac{\sum_{i}^{n}\left(f_{\theta, m i}-f_{\theta, c i}\right)}{n}
\end{aligned}
$$

where $k_{x}, k_{y}$ and $k_{\theta}$ are controller gains. $f_{u}$ and $f_{v}$ denote the image pixel coordinate of the mid point of the line segment, and $f_{\theta}$ denotes its orientation. $n$ is the number of the selected line features that are the extracted features of the chip. Index $i$ denotes feature number, and subscript $m$ and $c$ indicate the feature of the mark and the chip, 
respectively.

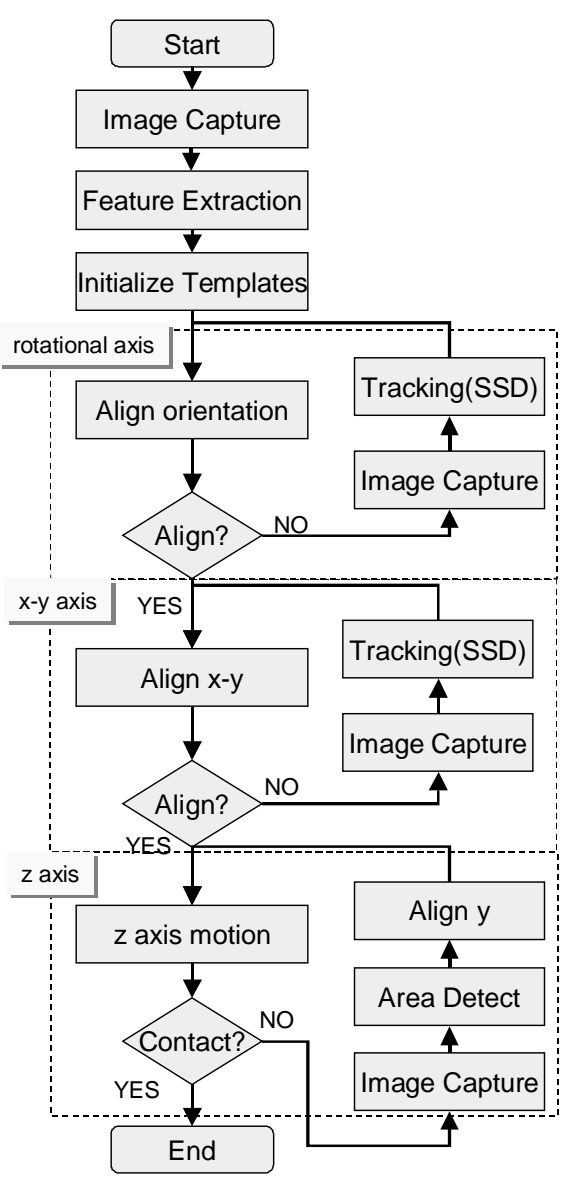

Figure 8: Flow Chart for Visual Servoing

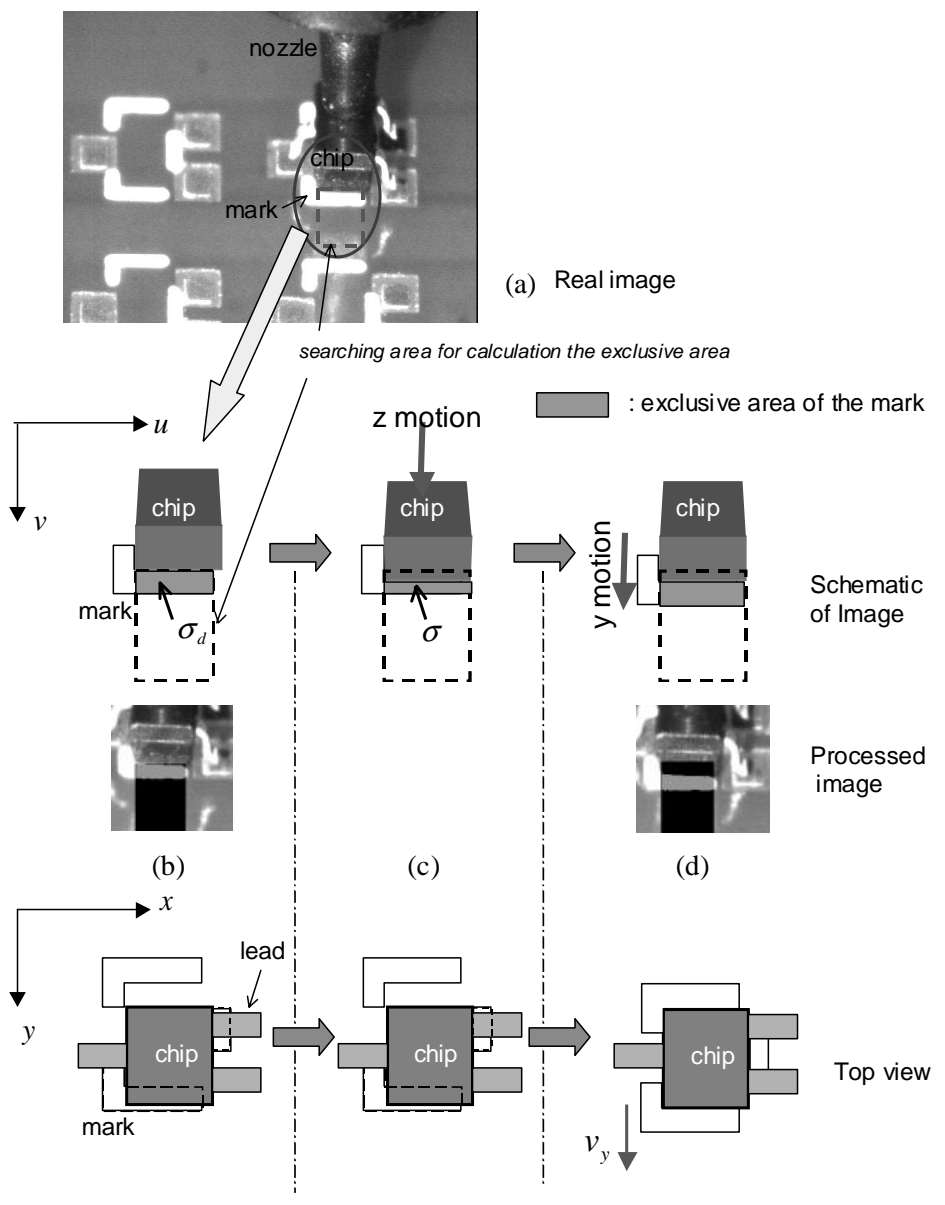

Figure 9: Servoing using Exclusive Area

\subsection{Visual servoing using exclusive area due to Occlusion}

After aligning in rotation and $\mathrm{x}-\mathrm{y}$ direction as Figure 9(a), the chip don't align with the mark on PCB in top view and the chip moves down, and the edge of the chip don't align with the inner boundary of the mark on the PCB board any longer as Figure 9(b). As the chip move down, the mark should move to direction keeping the alignment of them in order to align the edge of the chip with inner boundary of the mark. The stage moves to $y$ direction because the downward motion of the mounting head and $y$ motion of the stage correspond with the motion of $v$ axis on the image plane in our camera configuration. We define new feature, which represents the shown area of the mark occluded by the chip. This area is called an exclusive area because this area denotes mark's area excluded by the chip. Initially, exclusive area of the mark is saved as the desired are, $\sigma_{d}$. When the mounting head move down and the chip occludes the shown area of the mark, the proposed scheme generates the motion of $y$ axis as Figure 9(c) to keep up exclusive area of the mark by the chip in searching area. The following equation is applied for visual servoing

$$
v_{y}=k_{a}\left(\sigma_{d}-\sigma\right)
$$


where $k_{a}$ is a controller gain, and $\sigma$ denotes current exclusive area. The exclusive area is reduced, and then the stage move to $+y$ direction.

\subsection{Experimental Result}

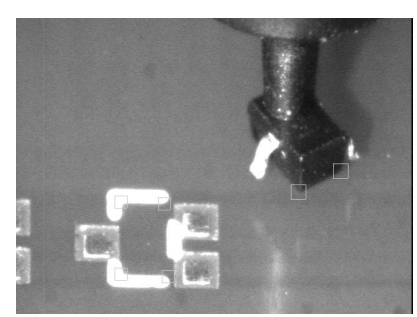

(a)

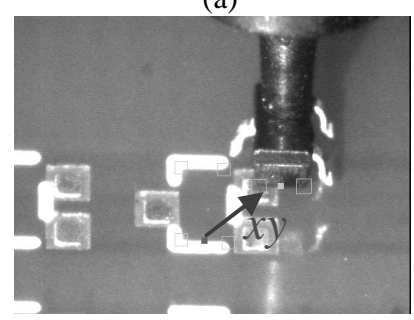

(d)

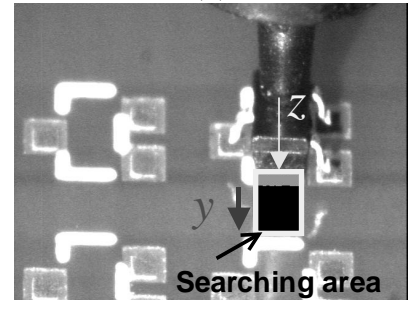

$(\mathrm{g})$

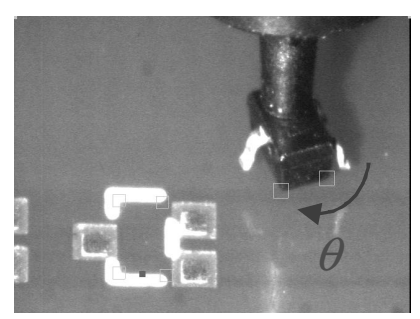

(b)

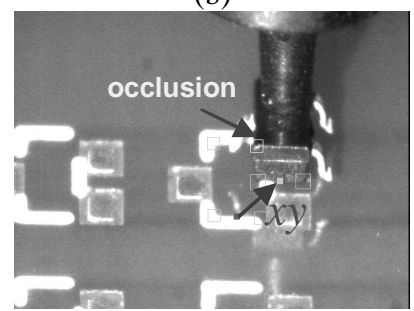

(e)

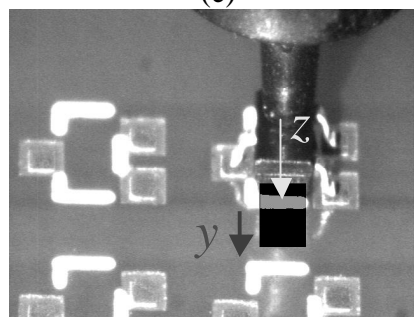

(h)

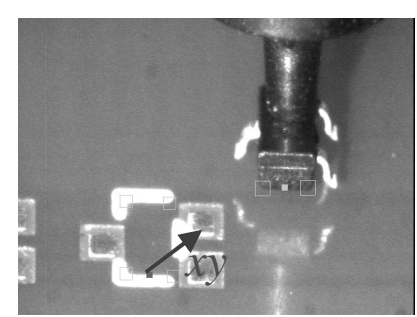

(c)

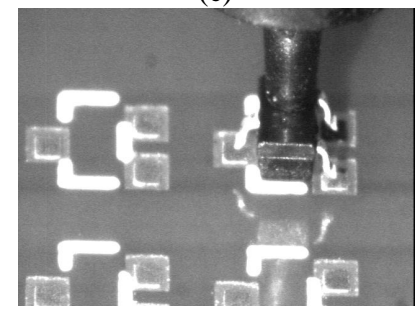

(f)

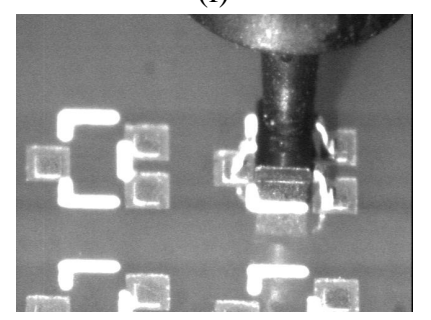

(i)

Figure 10: Experimental Result

Figure 10 shows the sequentially captured images when visual servoing scheme as shown in Figure 8 is applied to the proposed chip mounting. At first, the features are extracted, and then the templates are initialized for feature tracking as shown in Figure 10(a). The square represents the template at the feature point. The orientation of the chip is aligning with the mark by equation (16) as shown in Figure 10(b). After aligning the orientation, the mark approaches to the chip as shown in Figure 10(c)-(f). The chip occludes one of the features at Figure 10(e) and its position is estimated. In Figure (g)-(h), dark area represents the mark in searching area and black area represents the others. The number of dark pixel in searching area is the excusive area $\sigma$. As the chip moves down, the mark moves to y direction to keep the exclusive area $\sigma$ to the desired area $\sigma_{d}$, which represents the initial excusive area before moving down. Finally, the chip contacts with the PCB and the bottom edge of the chip are aligned the inner side of the mark.

\section{CONCLUSION}

In this paper, the chip mounting system with online feedback of the camera is proposed. This system introduces the occlusion problem at microscopic view. When the occlusion occurs during feature tracking, its position should be estimated by two clues. One of those is the geometry information of the features and the other is the motion information of the stage. The visual servoing using the mid point and the orientation of line segments and the 
exclusive area are applied. As the chip approaches to the mark on the PCB, the occluded feature is increased. In order to utilize many features, visual servoing scheme should make the part to approach the direction that reduces the number of occluded features or the occluded area until completing alignment of them. The sequential algorithm for visual servoing is applied to the chip mounting system, the real time experiment are perform to confirm the usefulness of the proposed algorithm. We plan to generalize the proposed approach in order to apply to precision assembly of a micro part.

\section{REFERENCES}

1. H. Yamamoto, A. Fujisatki and S. Kikuchi, "MCM and Bare Chip Technology For A Wide Range of Computers", Electronic Components and Technology Conference, pp. 133-138, Orlando, USA, 1996.

2. J. T. Feddenma and R. W. Simon, "CAD-Driven Microassembly and Visual Servoing”, Pro. of the 1998 IEEE Int. Conf. On Robotics and Automation, pp. 1212-1219, Leuven, Belgium, May, 1998.

3. S. J. Rais, B. Vikramaditya, and B. J. Nelson, "Micropositioning of a Weakly Calibrated Microassembly System Using Coarse-to-Fine Servoing Strategies", IEEE Transaction on Electronics Packaging Manufacturing, Vol. 23, No. 2, pp. 123-131, April, 2000.

4. Y. Zhou, B. J. Nelson and B. Vikramaditya, "Integrating Optical Force Sensing with Visual Servoing for Microassembly", Journal of Intelligent and Robotic Systems, No. 28, pp. 259-276, 2000.

5. N.R. Gans, P.I. Corke and S.A. Hutchinson, "Performance Test of Partitioned Approaches to Visual Servo Control", IEEE International Conference on Robotics \& Automation, pp. 1616-1623, USA, 2002.

6. E. Malis, F. Chaumette, and S. Boudet, "2-1/2-D Visual Servoing", IEEE Transactions on Robotics and Automation, Vol. 15, No. 2, pp. 238-250, 1999.

7. D.Y. Lee and H.S. Cho, "Precision Force Control via Macro/Micro Actuatorfor Surface Mounting System," IROS, Vol. 3, pp.2227-2232, EPFL Lausanne, Switzerland, 2002.

8. O. Faugeras, “Three-Dimensional Computer Vision - A Geometric Viewpoint”, The MIT Press, 2001. 